\title{
The influence of different head posture on occlusal contacts and its importance in restorative prosthodontics -A short term study
}

\author{
Shilpa Sinnurkar \\ Associate Professor, Dept. of Prosthodontics, ESIC Dental College and Hospital, Gulbarga, Karnataka, India
}

*Corresponding Author: Shilpa Sinnurkar

Email: drshilpa.s6@gmail.com

\begin{abstract}
Aim: To investigate the effect of sagittal plane head-neck posture on initial tooth contacts. Alteration of the position of the head will influence the neck and stomatognathic system, bringing the changes in occlusal stress distribution.

Objective: To evaluate the various head positions in an attempt to elucidate the head posture and occlusal contacts.

Materials and Methods: 20 subjects from 16 to 25 years of age with reasonable volunteer to participate in the study. The occlusal contacts are quantified by using occlusal indicator (okklean spray). Subjects with the head-neck in 450 dorsiflexion, 300 ventroflexion and in upright position. The head position is standardized by using pendulum goniometer.

Conclusion: Occlusal contacts position have significance in the analysis of dental health. The evaluation of the area and force of occlusal contact is helpful for diagnosing the occlusal state of patients. The selection of appropriate occlusal indicator for this purpose enables the dentist to work with precision and provides valuable information on occlusion refinement.
\end{abstract}

Keywords: Head position, occluasal contacts, ventroflexion, dorsiflexion and upright position.

\section{Introduction}

Head posture is a resultant response of environmental influences on the growth and development of the Craniomandibular complex. The importance of optimal head positioning is significant relative to many areas of dentistry such as bite registration, occlusal plane determination and occlusal management for fixed reconstruction, full denture, and orthodontic diagnosis and treatment. A physiologic level head position with an accompanying average 6-10 degree anterior sloping occlusal plane has been confirmed in literature. ${ }^{1}$

Occlusion is defined as the static relationship (contact) between the incising or masticating surfaces of the maxillary or mandibular teeth or tooth analogs. Head posture is a resultant response of environmental influences on the growth and development of the cranio-mandibular complex. ${ }^{2}$ The importance of optimal head positioning is significant relative to many areas of dentistry such as bite registration, occlusal plane determination and occlusal management for fixed reconstruction, full denture, and orthodontic diagnosis and treatment. Understanding the nature of contacts is important for the better diagnosis and treatment of stomatognathic diseases.

\section{Materials and Methods}

A convenience sample of 20 subjects (12 females \& 8 male) from 16 to 25 years of age were volunteered to participate in the study. A verbal consent was taken from all the subjects. The inclusion criteria for selection are as follows- young adults with permanent dentition except for the $3{ }^{\text {rd }}$ molar, sound periodontium and teeth without restoration and no history of Temporomandibular disorders and the exclusion criteria were patients with history of any restoration and orthodontic treatment, temporomandibular disorders and cervical spine diseases. With help of simple and accurate goniometer (an instrument for the precise measurement of angles, especially one used to measure the angles between the faces of crystals) a board was customized with three vertical extensions which indicates head position in three different angulation (i) In upright position (ii) $30^{\circ}$ ventroflexion and (iii) $45^{\circ}$ dorsoflexion which is attached to a stationary rigid wall (Fig. 1). Subject were comfortably seated with back support and vertical extension is attached over the head with the help of Velcro strap

\section{Procedure}

Patients mouth should be devoid of excess saliva. Head position is checked for the parallel to the floor and made seated on a chair with straight spinal position without any head rest. First one vertical extension is made stabilized over the head with the help of Velcro strap vertically when viewed on side profile and checked for the parallelism with the angulation on the board (Fig. 3). Occlusal indicator spray (Okklean spray DFC Diamon GmbH Riedenburg) (Fig. 2) is sprayed on the occlusal surface of maxillary arch and ask the patient to bite with minimal pressure in centric relation on articulating shim tracing paper to have initial light tooth contacts (Image 3 ). Record on tracing paper was checked for clear contacts on articulating shim paper and discard heavy occlusion with dispersed unclear contact. Procedure was repeated by changing the head position at 3 different angle that is at $45^{\circ}$ dorsoflexion (Fig. 4) and $30^{\circ}$ ventroflexion (Fig. 5) and upright position. The occlusal contacts were observed, counted on shim articulating paper and verified for clearance. Tabulated occlusal contacts were analyzed statistically.

\section{Results}

Tabulated occlusal contacts were subjected to paired $\mathrm{T}$ test for analysis.

1. Results shows significant difference ( $p<0.05$ ) among all the three angulation of the head position. 
2. More significant difference in upright to 30 degree ventroflexion (mesial contacts) as compared from that of upright to 45 degree dorsoflexion (distal contacts).

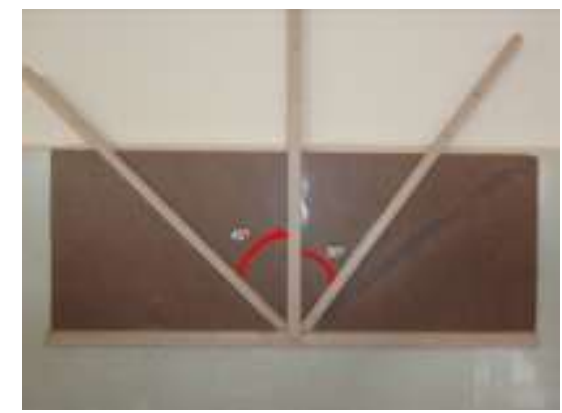

Fig. 1: Customized goniometer

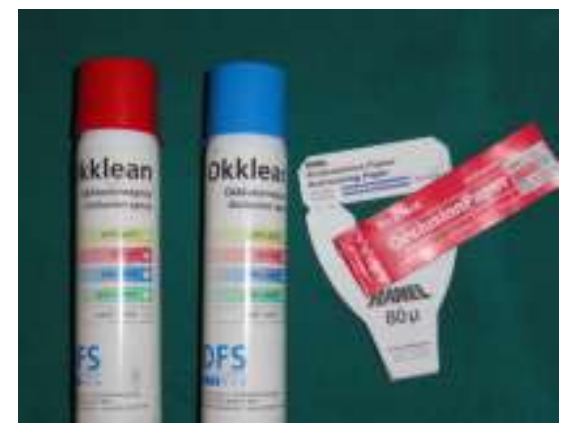

Fig. 2: Occlusal Indicators:- spray and articultaing papers

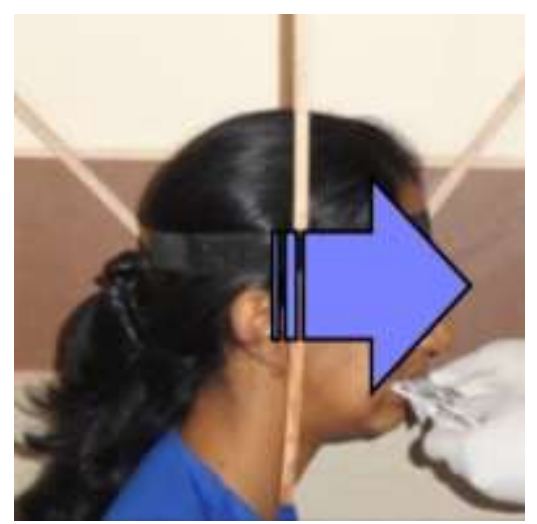

Fig. 3: Upright position

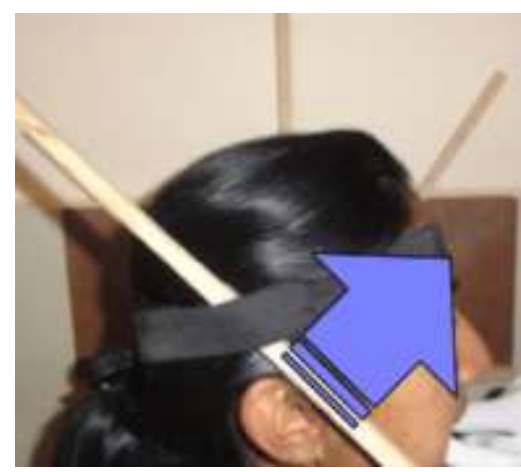

Fig. 4: $45^{0}$ dorsoflexion

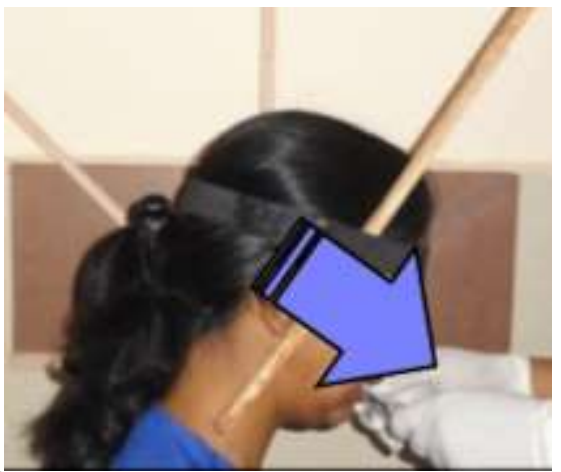

Fig. 5: $30^{\circ}$ ventroflexion

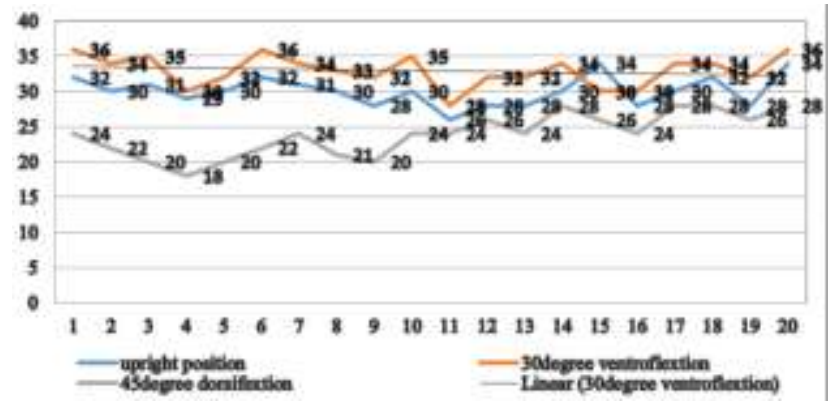

Fig. 6: Number of occlusal contants

\section{Discussion}

The reliability of recordings of dynamic occlusion also depends on the accuracy and reproducibility of repeated jaw movement, whereas consistent occlusal force is very difficult to control and may vary each day. From this result it appears that the frequency of changes in number occlusal contacts resulting from altering head position differs largely between subjects. On close observation of the study at 30 degree ventroflexion more number of mesial contacts were found from anterior region and at 45degree dorsoflexion, distal contacts were more from the posterior segment of the arch

Occlusal Interferences. Occlusal therapy aims at achieving simultaneous occlusal contact relationship. Any tooth contact that inhibits remaining occluding surface from achieving stable and harmonious contacts. True occlusal contact time $-0 \mathrm{sec}$ elapse. Any occlusal interference as small as 15 microns can trigger untoward response tooth pain radiating to head and neck region. True occlusal contact time that implies $0 \mathrm{sec}$ elapse between first and last contact. That implicates all the occlusal contact should meet simultaneously during mandibular closure ${ }^{3}$.

Occlusal indicators: Occlusal therapy aims at achieving simultaneous occlusal contact relationship. The selection of appropriate occlusal indicator for this purpose enables the dentist to work with precision and provides valuable information on occlusion refinement. (i) Qualitative occlusal Indicators: Qualitative indicators are most commonly used material for occlusal registration owing to their cost effect ant ease of application. With this material only localization of contact point is possible. Disadvantage is the sequence or density of contacts cannot be determined. Example- 
Articulating papers, Metallic shim stock film and High spot indicator. Articulating papers are most commonly used for registration in day to day practice. (ii) Quantitative occlusal Indicators: T- scan system and virtual patients are good example of quantitative and more reliable occlusal contacts. $\mathrm{T}$-scan identifies time magnitude and distribution of occlusal contacts. Virtual dental patient is recently introduced concept wherein $3 \mathrm{~d}$ dental patient is assembled from the data scanned from the cast of dentition ${ }^{3}$.

In 1973 McLean LF and Brenman HS demonstrated that tooth contact was different in supine and upright position following involuntary jaw closure elicited by electrical stimulation of the masseter muscle. Supine position results more retruded position of the mandible than upright position with mesial shift of the contacts ${ }^{4}$.

A study conducted by Etsuko Kondo, T.J. Aoba (1999) in two normally growing Japanese female patients with class II division 1 malocclusion presented with TMJ symptoms and poor head posture and as a result of abnormal sternocleidomastoid and trapezius cervical muscle activities. It was concluded that abnormal head posture can cause abnormal cervical muscle function and abnormal occlusion, adversely affecting the development and morphology of the cervical spine and maxillofacial skeleton, which in turn leads to facial asymmetry. ${ }^{5}$

\section{Limitations of the Study}

The study indicates a relationship between the head position and the number of occlusion contacts present. It is unknown to what extent this might induce modified therapeutic occlusal adjustment protocols. (b)Along with number of occlusal contacts, there was a linear relationship with other parameters occlusal force, occlusal contact area.

Clinical application. (a)Important during occlusal therapy procedure. (b)Bite registration. (c)occlusal plane determination. (d)Occlusal management from simple fixed prosthodontics to full mouth rehabilitation. (e)Orthodontic diagnosis and treatment.

\section{Conclusion}

Occlusal contacts position has been a significance in the analysis of dental health. The evaluation of the area and force of occlusal contact is helpful for diagnosing the occlusal state of patients. The selection of appropriate occlusal indicator for this purpose enables the dentist to work with precision and provides valuable information on occlusion refinement. Consequently, the present pilot study appears to be one of the few studies noted in literature that investigate the relationship between different head posture and number of occlusal contacts.

\section{Conflict of Interest: None.}

\section{References}

1. Howard Makofsky, Thomas R. Sexton, David diamond, Margherita t. Sexton. The effect of head posture on muscle contact position using the T-scan system of occlusal analysis. $J$ Craniomandibular Pract 1991;9(4):316-21

2. Takahisa Shimazaki, Mitsuru Motoyoshi, Koheihosoi and Shinkichi Namura.The effect of occlusal alteration and masticatory imbalance on the cervical spine. Eur $J$ Orthodontics 2003;25:457-63.

3. Ramakrishna Rajan, Nayar Sanjna Vibhu. Occlusion indicators: A review. J Indian Prosthodont Soc 2007;7(4):17074.

4. McLean LF, Brenman HS, Friedman MG. Effect of body position on dental occlusion. J Dent Res 1973;52(5):1041-5.

5. Etsuko Kondo, T J Aoba. Case report of malocclusion with abnormal head posture and TMJ symptoms. Am J Orthod Dent Orthop 1999;116(5):481-93.

6. Akimoto S, Fushima k, Sato S, Suzukiy. Masticatory muscle activity of facial asymmetry cases - effects of the different combination of the bite blocks. J Japanese Orthod Soc 1994;53:632-40.

7. Macdonald JW, Hannam. A relationship between occlusal. Contacts and jaw-closing muscle activity during tooth clenching: Part I. J Prosthet dent 1984;52:718-28.

8. Salonen m, Raustia a, Huggare J. Changes in head and cervical spine posture and EMG activities of masticatory muscles following treatment with complete upper and partial lower dentures. J Craniomandibular Pract 1994;12:222-6.

9. Özbek m, köklü. A natural cervical inclination and craniofacial structure. Am J Orthod Dent Orthop 1993;104:584-91

10. Visscher $\mathrm{cm}$, Huddleston slater JJ. Kinematics of the human mandible for different head posture. J Oral Rehabil 2000;27: 299-305.

How to cite this article: Sinnurkar S. The influence of different head posture on occlusal contacts and its importance in restorative prosthodontics -A short term study. Ann Prosthodont Restor Dent 2019;5(2):50-52. 